PROCEEDINGS OF THE

AMERICAN MATHEMATICAL SOCIETY

Volume 134, Number 6, Pages 1651-1660

S 0002-9939(05)08329-2

Article electronically published on October 18,2005

\title{
ALMOST EVERYWHERE CONVERGENCE OF INVERSE FOURIER TRANSFORMS
}

\author{
LEONARDO COLZANI, CHRISTOPHER MEANEY, AND ELENA PRESTINI \\ (Communicated by Andreas Seeger)
}

\begin{abstract}
We show that if $\log (2-\Delta) f \in L^{2}\left(\mathbb{R}^{d}\right)$, then the inverse Fourier transform of $f$ converges almost everywhere. Here the partial integrals in the Fourier inversion formula come from dilates of a closed bounded neighbourhood of the origin which is star shaped with respect to 0 . Our proof is based on a simple application of the Rademacher-Menshov Theorem. In the special case of spherical partial integrals, the theorem was proved by Carbery and Soria. We obtain some partial results when $\sqrt{\log (2-\Delta)} f \in L^{2}\left(\mathbb{R}^{d}\right)$ and $\log \log (4-\Delta) f \in L^{2}\left(\mathbb{R}^{d}\right)$. We also consider sequential convergence for general elements of $L^{2}\left(\mathbb{R}^{d}\right)$.
\end{abstract}

\section{INTRODUCTION}

We treat the almost everywhere convergence of partial integrals of inverse Fourier transforms on Euclidean space, for functions in $L^{2}$ with logarithmic Sobolev properties. The partial integrals are formed by integrating over dilates of a fixed closed bounded region $V$ which is star shaped with respect to the origin and has the origin in its interior. Particular choices of $V$ give rise to the familiar cases of spherical and polyhedral partial integrals. Our results are proved by a very simple application of the Rademacher-Menshov Theorem. In particular, we show that if the Fourier transform satisfies

$$
\int_{\mathbb{R}^{d}}\left(\log \left(2+|y|^{2}\right)\right)^{2}|\widehat{f}(y)|^{2} d y<\infty,
$$

then the partial integrals

$$
S_{R} f(x)=\int_{R V} \widehat{f}(y) e^{2 \pi i x \cdot y} d y
$$

Received by the editors December 27, 2004

2000 Mathematics Subject Classification. Primary 42B10, 43A50; Secondary 42C15.

Key words and phrases. Rademacher-Menshov Theorem, inverse Fourier transform, series of orthogonal functions.

The second author was partially supported by Progetto cofinanziato MIUR "Analisi Armonica". We are grateful to Fulvio Ricci and the Centro di Ricerca Matematica Ennio De Giorgi for their hospitality.

The third author was partially supported by Progetto cofinanziato MIUR "Analisi Armonica".

(C)2005 American Mathematical Society Reverts to public domain 28 years from publication 
converge almost everywhere as $R \rightarrow \infty$. If we reduce the power of the logarithmic factor, we have a partial result. We show that if

$$
\int_{\mathbb{R}^{d}} \log \left(2+|y|^{2}\right)|\widehat{f}(y)|^{2} d y<\infty,
$$

then $S_{R_{n}} f(x)$ converges almost everywhere as $R_{n}=n^{\log (n)} \rightarrow \infty$. When the logarithm is replaced by $\log \log$, we find that if

$$
\int_{\mathbb{R}^{d}}\left(\log \log \left(4+|y|^{2}\right)\right)^{2}|\widehat{f}(y)|^{2} d y<\infty
$$

then $S_{r_{m}} f(x)$ converges almost everywhere as $m \rightarrow \infty$, for unbounded sequences $\left(r_{m}\right)$ whose terms are in a second order lacunary set, as defined in 2 .

The first case, with $V$ a sphere, was done by Carbery and Soria [4, Theorem 3]. The introduction to their paper provides a broad description to the background of this area of Fourier analysis. See also [6 for some weighted norm estimates in the spherical case. The third case is a slight extension of the main result in [2], where they work with integrals over spheres.

Our contribution is the simplicity of the proof and the fact that it is independent of the geometry of $V$. The method seems to depend only on the Plancherel formula, and follows the same idea as used in 8 .

\section{The Rademacher-Menshov Theorem}

Theorem 1. Suppose that $(X, \mu)$ is a positive measure space. There is a positive constant $c$ with the following property.

For each orthogonal subset $\left\{P_{n}: n \in \mathbb{N}\right\}$ in $L^{2}(X, \mu)$ which satisfies

$$
\sum_{n=1}^{\infty}(\log (n+1))^{2}\left\|P_{n}\right\|_{2}^{2}<\infty
$$

the maximal function $\mathcal{M}(x)=\sup _{N \geq 1}\left|\sum_{n=1}^{N} P_{n}(x)\right|$ is in $L^{2}(X, \mu)$ and

$$
\|\mathcal{M}\|_{2} \leq c\left(\sum_{n=1}^{\infty}(\log (n+1))^{2}\left\|P_{n}\right\|_{2}^{2}\right)^{1 / 2} .
$$

In particular, when (4) holds, then the series $\sum_{n=1}^{\infty} P_{n}(x)$ converges almost everywhere on $X$.

See Theorem XIII.10.21 from [11, Proposition 2.3.1, and Theorem 2.3.2 from [1, Pages 79-80]. Here log means logarithm with base 2. For an application in $L^{2}\left(\mathbb{R}^{d}\right)$, see part (b) of Lemma 5.1 in 5 .

\section{Setting up the Partial Integrals}

Suppose that $V$ is a bounded closed subset of $\mathbb{R}^{d}$ having 0 as an interior point and star shaped with respect to 0 . Let $\beta=d(0, \partial V)>0$. For each $R>0$ dilate $V$ to get $R V=\{R y: y \in V\}$, so that the dilated set has measure

$$
|R V|=R^{d}|V| \quad \text { and } \quad d(0, \partial(R V))=R \beta .
$$

Define partial integrals by

$$
S_{R} f(x)=\int_{R V} \widehat{f}(y) e^{2 \pi i x \cdot y} d y, \quad \forall f \in L^{2}\left(\mathbb{R}^{d}\right),
$$


which give Fourier inversion in norm, $\lim _{R \rightarrow \infty}\left\|S_{R} f-f\right\|_{2}=0$. If $f \in L^{2}\left(\mathbb{R}^{d}\right)$ and $S_{R} f(x)$ converges almost everywhere as $R \rightarrow \infty$, then its limit equals $f(x)$ almost everywhere.

Now let $\left(R_{n}\right)_{n=1}^{\infty}$ be an unbounded increasing sequence of positive real numbers and fix an element $f \in L^{2}\left(\mathbb{R}^{d}\right)$. We think of the partial integrals $S_{R_{n}} f(x)$ as partial sums of the orthogonal expansion

$$
S_{R_{1}} f(x)+\sum_{n=2}^{\infty}\left(S_{R_{n}} f(x)-S_{R_{n-1}} f(x)\right) .
$$

Define $P_{n} f \in L^{2}\left(\mathbb{R}^{d}\right)$ by setting

$$
P_{n} f(x)= \begin{cases}S_{R_{1}} f(x) & \text { if } n=1, \\ S_{R_{n}} f(x)-S_{R_{n-1}} f(x) & \text { if } n \geq 2 .\end{cases}
$$

Then the partial sums of (7) are

$$
S_{R_{n}} f(x)=\sum_{k=1}^{n} P_{k} f(x), \quad \forall n \geq 1, x \in \mathbb{R}^{d},
$$

and $m \neq n$ implies that $P_{m} f \perp P_{n} f$. The Plancherel formula says that

$$
\left\|P_{n} f\right\|_{2}^{2}=\int_{R_{n} V \backslash R_{n-1} V}|\widehat{f}(y)|^{2} d y .
$$

\section{Convergent subsequences}

Suppose that $f \in L^{2}\left(\mathbb{R}^{d}\right)$. Since $S_{R} f$ converges to $f$ in norm, there exists a sequence $\left(R_{n}\right)_{n=0}^{\infty}$ with $\lim _{n \rightarrow \infty} S_{R_{n}} f(x)=f(x)$ almost everywhere. The RademacherMenshov Theorem gives a way of describing one such sequence.

Proposition 2. Suppose $f \in L^{2}\left(\mathbb{R}^{d}\right)$. If an increasing unbounded sequence $0=$ $R_{0}<R_{1}<R_{2}<\cdots$ has the property

$$
\sum_{n=1}^{\infty}\left\|S_{R_{n}} f-S_{R_{n-1}} f\right\|_{2}^{2}(\log (n+1))^{2}<\infty
$$

then $\lim _{n \rightarrow \infty} S_{R_{n}} f(x)=f(x)$ almost everywhere. Furthermore, for each $f \in$ $L^{2}\left(\mathbb{R}^{d}\right)$ there is an increasing unbounded sequence $\left(R_{n}\right)_{n=0}^{\infty}$ with property (10).

Proof. The first statement is a direct consequence of Theorem 1 It remains to prove the second statement. If $\widehat{f}$ has bounded support, then it is integrable and the statement is immediate. Now suppose that $\widehat{f}$ is not compactly supported. The function $R \mapsto F(R)=\left\|S_{R} f\right\|_{2}$ is continuous and its values are non-negative. If $R^{\prime}<R^{\prime \prime}$, then $F\left(R^{\prime}\right) \leq F\left(R^{\prime \prime}\right)$, and $\lim _{R \rightarrow \infty} F(R)=\|f\|_{2}$. Let $\left(a_{n}\right)_{n=1}^{\infty}$ be a sequence of positive numbers with

$$
\sum_{n=1}^{\infty} a_{n}=1 \quad \text { and } \quad \sum_{n=1}^{\infty}(\log (n+1))^{2} a_{n}<\infty .
$$

There is an increasing unbounded sequence $\left(R_{n}\right)_{n=1}^{\infty}$ with the property

$$
F\left(R_{n}\right)^{2}=\|f\|_{2}^{2} \sum_{m=1}^{n} a_{m}, \quad \forall n \geq 1 .
$$


In particular, $\left\|S_{R_{n+1}} f\right\|_{2}^{2}-\left\|S_{R_{n}} f\right\|_{2}^{2}=a_{n+1}\|f\|_{2}^{2}$, for all $n \geq 1$. Define the projections as in (8). Then we have that

$$
\sum_{n=1}^{\infty}(\log (n+1))^{2}\left\|P_{n} f\right\|_{2}^{2}<\infty
$$

and we can apply Theorem 1 .

The Cauchy-Schwarz inequality and the Plancherel formula imply that when a sequence of partial integrals converges, then the sequence can be perturbed slightly and still preserve convergence.

Lemma 3. Suppose $\left(R_{n}\right)_{n=1}^{\infty}$ is an increasing unbounded sequence. For each $\rho>0$ and $n \geq 1$ define the set

$$
E_{\rho}(n)=\left\{r>0:\left|r^{d}-R_{n}^{d}\right| \leq \rho\right\} .
$$

For these sets and $f \in L^{2}\left(\mathbb{R}^{d}\right)$ there is the inequality,

$$
\sup _{n \geq 1}\left(\sup _{r \in E_{\rho}(n)}\left|S_{r} f(x)-S_{R_{n}} f(x)\right|\right) \leq\|f\|_{2} \sqrt{\rho|V|}, \quad \forall x \in \mathbb{R}^{d} .
$$

Now fix $f \in L^{2}\left(\mathbb{R}^{d}\right)$ and suppose $\left(R_{n}\right)_{n=1}^{\infty}$ is an increasing unbounded sequence for which $S_{R_{n}} f(x)$ converges almost everywhere. Furthermore, let $E_{\rho}=\bigcup_{n=1}^{\infty} E_{\rho}(n)$. If $\left(r_{m}\right)_{m=1}^{\infty}$ is an increasing unbounded sequence whose terms belong to a set $E_{\rho}$, then $\lim _{m \rightarrow \infty} S_{r_{m}} f(x)=f(x)$, almost everywhere.

Proof. If $0 \leq R_{n}^{d}-r^{d} \leq \rho$, then $r V \subset R_{n} V$ and $\left|R_{n} V \backslash r V\right| \leq \rho|V|$, so that

$$
\left|S_{R_{n}} f(x)-S_{r} f(x)\right| \leq\left(\int_{R_{n} V \backslash r V}|\widehat{f}(y)|^{2} d y\right)^{1 / 2} \sqrt{\rho|V|} .
$$

Since $f \in L^{2}\left(\mathbb{R}^{d}\right)$, the right-hand side tends to zero as $R_{n} \rightarrow \infty$. A similar argument applies to the case $0 \leq r^{d}-R_{n}^{d} \leq \rho$.

We can apply the Rademacher-Menshov Theorem again to give a minor extension of Proposition 2 ,

Lemma 4. Suppose that $f \in L^{2}\left(\mathbb{R}^{d}\right)$ and $\left(R_{n}\right)_{n=0}^{\infty}$ satisfy (10) of Proposition 2 . If $\left(r_{m}\right)_{m=1}^{\infty}$ is an unbounded increasing sequence with the property that

$$
\left|\left\{m: R_{n} \leq r_{m} \leq R_{n+1}\right\}\right| \leq c n^{\gamma}, \quad \forall n \geq 1,
$$

for some positive constants $c$ and $\gamma$, then

$$
\lim _{m \rightarrow \infty} S_{r_{m}} f(x)=f(x), \quad \text { almost everywhere. }
$$

Proof. For each $n \geq 1$, suppose that there is a finite set of $M_{n}$ real numbers arranged in the interval $\left(R_{n}, R_{n+1}\right)$, say

$$
R_{n}=r_{1}(n)<\cdots<r_{M_{n}}(n)=R_{n+1}
$$

and define functions

$$
Q_{k, n}(x)=S_{r_{k+1}(n)} f(x)-S_{r_{k}(n)} f(x), \quad 1 \leq k<M_{n} .
$$


These functions form an orthogonal subset of $L^{2}\left(\mathbb{R}^{d}\right)$ and so the RademacherMenshov Theorem says that

$$
\max _{1 \leq m<M_{n}}\left|S_{r_{m}(n)} f(x)-S_{R_{n}} f(x)\right|=\max _{1 \leq m<M_{n}}\left|\sum_{k=1}^{m} Q_{k, n}(x)\right|
$$

has $L^{2}$ norm bounded by

$$
c\left(\log M_{n}\right)\left\|S_{R_{n+1}} f-S_{R_{n}} f\right\|_{2}=c\left(\log M_{n}\right)\left\|P_{n+1} f\right\|_{2} .
$$

Suppose that

$$
\log M_{n} \leq \gamma \log n=\log \left(n^{\gamma}\right), \quad \forall n \geq 2 .
$$

Because of (10) we see that

$$
\sum_{n=1}^{\infty} \max _{1 \leq m \leq M_{n}}\left|S_{r_{m}(n)} f(x)-S_{R_{n}} f(x)\right|^{2}
$$

is in $L^{1}\left(\mathbb{R}^{d}\right)$. We then have that as $n \rightarrow \infty$,

$$
\max _{1 \leq m \leq M_{n}}\left|S_{r_{m}(n)} f(x)-S_{R_{n}} f(x)\right| \rightarrow 0, \text { almost everywhere. }
$$

\section{The MAIn RESUlt}

Proposition 5. Suppose that $f \in L^{2}\left(\mathbb{R}^{d}\right)$ satisfies the condition (11). Then $\lim _{R \rightarrow \infty} S_{R} f(x)=f(x)$, almost everywhere on $\mathbb{R}^{d}$. Furthermore, there is a constant $c>0$ so that for all $w \in \mathbb{R}^{d}$,

$$
\int_{|x-w| \leq 1}\left|\sup _{R>0}\right| S_{R} f(x)||^{2} d x \leq c \int_{\mathbb{R}^{d}}\left(\log \left(2+|y|^{2}\right)\right)^{2}|\widehat{f}(y)|^{2} d y .
$$

Proof. Take the sequence $R_{n}=n^{1 / d}$ in setting up (8) and let

$$
\mathcal{M} f(x)=\sup _{n \geq 1}\left|S_{R_{n}} f(x)\right|, \quad \forall x \in \mathbb{R}^{d} .
$$

When $y$ is in the shell $R_{n} V \backslash R_{n-1} V$ it satisfies $|y| \geq(n-1)^{1 / d} \beta$ and for large $n$ there is a constant $c>0$ for which

$$
\log (n+1) \leq c \log \left(2+|y|^{2}\right), \quad \forall y \in R_{n} V \backslash R_{n-1} V
$$

Combine this with (9) to see that

$$
(\log (n+1))^{2}\left\|P_{n}\right\|_{2}^{2} \leq c \int_{R_{n} V \backslash R_{n-1} V}\left(\log \left(2+|y|^{2}\right)\right)^{2}|\widehat{f}(y)|^{2} d y .
$$

Since $f$ satisfies inequality (1), the sum of the terms on the right-hand side is finite. This verifies the hypothesis (4) in Theorem 1 and so $S_{R_{n}} f(x)$ converges almost everywhere as $n \rightarrow \infty$. Furthermore, we see that since (11) holds, then inequality (5) says that

$$
\|\mathcal{M} f\|_{2} \leq c\left(\int_{\mathbb{R}^{d}}\left(\log \left(2+|y|^{2}\right)\right)^{2}|\widehat{f}(y)|^{2} d y\right)^{1 / 2}
$$


We can dominate the maximal function over $R \geq 1$ by the maximal function over the sequence $\left(R_{n}\right)_{n=1}^{\infty}$ plus a remainder,

$$
\sup _{R \geq 1}\left|S_{R} f(x)\right| \leq \mathcal{M} f(x)+\sup _{n>0}\left(\sup _{R_{n} \leq r<R_{n+1}}\left|S_{r} f(x)-S_{R_{n}} f(x)\right|\right) .
$$

We chose the sequence $R_{n}=n^{1 / d}$ so that the increments in the measure of the dilates of $V$ are constant,

$$
\left|R_{n} V \backslash R_{n-1} V\right|=n|V|-(n-1)|V|=|V| .
$$

If $R_{n} \leq r<R_{n+1}$, then $n \leq r^{d}<n+1$ and $\left|r^{d}-n\right|=\left|r^{d}-R_{n}^{d}\right| \leq 1$, so that we can apply Lemma 3 with $\rho=1$. Hence

$$
\left\|\sup _{n>0}\left(\sup _{R_{n} \leq r<R_{n+1}}\left|S_{r} f-S_{R_{n}} f\right|\right)\right\|_{\infty} \leq c\|f\|_{2} .
$$

Combine inequalities (13) and (14) to prove (12).

See [6, Chapter 2] for more sophisticated methods for estimating $S_{R_{n}} f(x)-$ $S_{r} f(x)$.

\section{The CASE OF ONE POWER OF LOGARITHM}

The first part of the method used above can be applied to other sequences.

Proposition 6. Suppose that $f \in L^{2}\left(\mathbb{R}^{d}\right)$ satisifies the condition (21) and that $R_{n}=n^{\log n}$, for $n \geq 1$. Then $\lim _{n \rightarrow \infty} S_{R_{n}} f(x)=f(x)$, almost everywhere on $\mathbb{R}^{d}$.

Proof. We have that $\log \left(R_{n}\right)=(\log n)^{2}$ and for large $n$ there is a constant $c$ for which

$$
(\log (n+1))^{2}\left\|P_{n} f\right\|_{2}^{2} \leq c \int_{R_{n} V \backslash R_{n-1} V} \log \left(2+|y|^{2}\right)|\widehat{f}(y)|^{2} d y .
$$

Inequality (2) means that the sum of the terms on the right-hand side is finite and so Theorem 1 applies.

Note that $n^{\log n}=2^{(\log n)^{2}}$ grows slower than any unbounded geometric progression but faster than $n^{k}$, for each $k \in \mathbb{N}$. The measure of the shell $R_{n} V \backslash R_{n-1} V$ grows too rapidly to use the estimate from Lemma 3 . However, Lemma 4 gives convergence for some other sequences.

Corollary 7. Suppose that $f \in L^{2}\left(\mathbb{R}^{d}\right)$ satisfies (2) and $\left(r_{m}\right)_{m=1}^{\infty}$ is an unbounded increasing sequence with the property that

$$
\left|\left\{m: n^{\log n} \leq r_{m} \leq(n+1)^{\log (n+1)}\right\}\right| \leq c n^{\gamma}, \quad \forall n \geq 1,
$$

for some positive constants $c$ and $\gamma$. Then $\lim _{m \rightarrow \infty} S_{r_{m}} f(x)=f(x)$, almost everywhere. 


\section{ITERATED LOGARITHM}

Fix $a>1$ and define the geometric progression $R_{n}=a^{n}$, for all $n \geq 1$. For $y \in R_{n} V \backslash R_{n-1} V$ we have $|y| \geq a^{n-1} \beta$ and for large $n$ there is a constant $\kappa>0$ with

$$
\log \log \left(4+|y|^{2}\right) \geq \kappa \log (n+1) .
$$

This means that for large $n$ we have

$$
\begin{aligned}
\kappa^{2}(\log (n+1))^{2} \int_{R_{n} V \backslash R_{n-1} V}|\widehat{f}(y)|^{2} d y & \\
& \leq \int_{R_{n} V \backslash R_{n-1} V}\left(\log \log \left(4+|y|^{2}\right)\right)^{2}|\widehat{f}(y)|^{2} d y
\end{aligned}
$$

and we can again apply Theorem 1 .

Corollary 8. Suppose that $f \in L^{2}\left(\mathbb{R}^{d}\right)$ satisifies (3) and that $a>1$ is fixed. Then $\lim _{n \rightarrow \infty} S_{a^{n}} f(x)=f(x)$, almost everywhere on $\mathbb{R}^{d}$.

Remark 7.1. For lacunary spherical partial integrals there is a much stronger result in [3, Theorem B] and in [7].

Lemma 4 can be applied to the case of $R_{n}=a^{n}$.

Corollary 9. Fix $a>1$ and let $\left(r_{m}\right)_{m=1}^{\infty}$ be an unbounded increasing sequence with the property that

$$
\left|\left\{m: a^{n} \leq r_{m} \leq a^{n+1}\right\}\right| \leq c n^{\gamma}, \quad \forall n \geq 1
$$

for some positive constants $c$ and $\gamma$. If $f \in L^{2}\left(\mathbb{R}^{d}\right)$ satisfies (3), then

$$
\lim _{m \rightarrow \infty} S_{r_{m}} f(x)=f(x), \quad \text { almost everywhere. }
$$

We can combine Corollary 8 with Lemma 3 and Corollary 9 to extend the result of [2] to the case of general $V$.

Corollary 10. Fix $a>1$ and suppose $f \in L^{2}\left(\mathbb{R}^{d}\right)$ satisfies (3). Let

$$
A=\left\{a^{n}\left(1-a^{-k}\right): n, k \in \mathbb{N}\right\}
$$

and suppose that $\left(r_{m}\right)_{m=1}^{\infty}$ is an increasing unbounded sequence whose terms belong to $A$. Then $\lim _{m \rightarrow \infty} S_{r_{m}} f(x)=f(x)$, almost everywhere.

Proof. Let $R_{n}=a^{n}$ and consider the set $E_{1}$, as defined in Lemma 3 . We need to count how many elements are in $\left(A \backslash E_{1}\right) \cap\left[a^{n-1}, a^{n}\right]$, for each $n \geq 1$. That is, we count how many $k$ satisfy

$$
a^{n d}-a^{n d}\left(1-a^{-k}\right)^{d}>1
$$

This is equivalent to the inequality

$$
1-\left(1-a^{-k}\right)^{d}>a^{-d n}
$$

and the left-hand side is equal to $d a^{-k} y^{d-1}$ for some $1-a^{-k} \leq y \leq 1$. Taking logarithms, we see that if $k$ satisfies the inequality (16), then we must have $k \leq c n$, for some constants $c$. This shows that $A \backslash E_{1}$ satisfies the criterion of Corollary 9 . If a sequence has its values in $A$, then it is made up of subsequences in $A \cap E_{1}$ and $A \backslash E_{1}$. Apply Lemma 3 for $A \cap E_{1}$ and Corollary 9 for $A \backslash E_{1}$. 


\section{Capacity}

We conclude with an extension of Theorem 1.3 of [9] to summation based on the set $V$. Following Definition 2 in [9], for each $0<\alpha<d$ the $(\alpha, 2)$-capacity of a subset $X \subset \mathbb{R}^{d}$ is

$$
C_{\alpha}(X)=\inf \left\{\|f\|_{2}^{2}: f \in L_{+}^{2}\left(\mathbb{R}^{d}\right), \quad G_{\alpha} * f(x) \geq 1, \forall x \in X\right\} .
$$

Here $G_{\alpha}$ is the Bessel kernel, with $\widehat{G_{\alpha}}(y)=\left(1+|y|^{2}\right)^{-\alpha / 2}$. Its properties are cataloged in [10, Section V.3]. Most importantly, $G_{\alpha}(x) \geq 0$ for all $x \neq 0$. Notice that if $f \in L_{+}^{2}\left(\mathbb{R}^{d}\right)$ and $X \subseteq\left\{x: G_{\alpha} * f(x) \geq \lambda\right\}$, then $C_{\alpha}(X) \leq \lambda^{-2}\|f\|_{2}^{2}$. Capacity is subadditive and sets of capacity zero have Lebesgue measure zero.

Let $R_{n}=n^{1 / d}$ for each $n \geq 1$, as in the proof of Proposition 5, and define $\mathcal{M} f(x)=\sup _{n \geq 1}\left|S_{R_{n}} f(x)\right|$. Recall that this satisfies inequality (13).

Lemma 11. Suppose that $\varphi \in L^{2}\left(\mathbb{R}^{d}\right)$ satisfies

$$
N(\varphi, \alpha):=\int_{\mathbb{R}^{d}}|\widehat{\varphi}(y)|^{2}\left(1+|y|^{2}\right)^{\alpha}(\log (2+|y|))^{2} d y<\infty,
$$

for some $0<\alpha<d$. There is a positive constant $c$ so that

$$
C_{\alpha}(\{x: \mathcal{M} \varphi(x) \geq \lambda\}) \leq c \lambda^{-2} N(\varphi, \alpha), \quad \forall \lambda>0 .
$$

Proof. Since $\varphi$ satisfies (17), there is a $\psi \in L^{2}\left(\mathbb{R}^{d}\right)$ with $\varphi=G_{\alpha} * \psi$ and

$$
N(\varphi, \alpha)=N(\psi, 0)=\int_{\mathbb{R}^{d}}|\widehat{\psi}(y)|^{2}(\log (2+|y|))^{2} d y<\infty .
$$

Inequality (13) can be applied to both $\psi$ and to $\varphi=G_{\alpha} * \psi$, so that the maximal functions satisfy $\mathcal{M} \psi \in L^{2}\left(\mathbb{R}^{d}\right)$ and $\mathcal{M}\left(G_{\alpha} * \psi\right) \in L^{2}\left(\mathbb{R}^{d}\right)$. Since $G_{\alpha}$ is positive, the observation on page 1419 of [9] can be adapted to our sequential maximal function so that

$$
\mathcal{M}\left(G_{\alpha} * \psi\right)(x) \leq G_{\alpha} *(\mathcal{M} \psi)(x), \quad \forall x \in \mathbb{R}^{d}
$$

For each $\lambda>0$ let

$$
X_{\lambda}=\left\{x: \mathcal{M}\left(G_{\alpha} * \psi\right)(x) \geq \lambda\right\} \subseteq\left\{x: G_{\alpha} *(\mathcal{M} \psi)(x) \geq \lambda\right\} .
$$

From the definition of capacity, $C_{\alpha}\left(X_{\lambda}\right) \leq \lambda^{-2}\|\mathcal{M} \psi\|_{2}^{2} \leq c \lambda^{-2} N(\psi, 0)$.

Proposition 12. Suppose that $\varphi \in L^{2}\left(\mathbb{R}^{d}\right)$ satisfies (17) for some $0<\alpha<d$. The set on which $S_{R} \varphi(x)$ does not converge to $\varphi(x)$, as $R \rightarrow \infty$, has $(\alpha, 2)$-capacity zero.

Proof. The argument based on Lemma 3 shows that it is enough to consider the convergence of $S_{R_{n}} \varphi(x)$ as $n \rightarrow \infty$. Let $\psi$ be the function in the previous proof, so that $\varphi=G_{\alpha} * \psi$. For $\delta>0$ let $H \in C_{c}^{\infty}\left(\mathbb{R}^{d}\right)$ satisfy

$$
N(\psi-H, 0)=\int_{\mathbb{R}^{d}}|\widehat{\psi}(y)-\widehat{H}(y)|^{2}(\log (2+|y|))^{2} d y<\delta .
$$

We know that $\lim _{R \rightarrow \infty} S_{R}\left(G_{\alpha} * H\right)(x)=G_{\alpha} * H(x)$, for all $x$. For each $\eta>0$,

$$
\begin{gathered}
\left\{x: \limsup _{n \rightarrow \infty}\left|S_{R_{n}} \varphi(x)-\varphi(x)\right|>\eta\right\} \\
\subseteq\left\{x: \sup _{n \geq 1}\left|S_{R_{n}}\left(\varphi-G_{\alpha} * H\right)(x)\right|>\frac{\eta}{2}\right\} \cup\left\{x:\left|\varphi(x)-G_{\alpha} * H(x)\right|>\frac{\eta}{2}\right\} .
\end{gathered}
$$


Lemma 11 shows that

$$
C_{\alpha}\left(\left\{x: \sup _{n \geq 1}\left|S_{R_{n}}\left(G_{\alpha} * \psi-G_{\alpha} * H\right)(x)\right|>\frac{\eta}{2}\right\}\right) \leq 4 c \eta^{-2} \delta .
$$

Observe that $\left|G_{\alpha} * \psi-G_{\alpha} * H\right| \leq G_{\alpha} *|\psi-H|$. The definition of capacity shows that

$$
C_{\alpha}\left(\left\{x:\left|G_{\alpha} * \psi(x)-G_{\alpha} * H(x)\right|>\frac{\eta}{2}\right\}\right) \leq 4 \eta^{-2}\|\psi-H\|_{2}^{2}<4 \eta^{-2} c_{d}^{2} \delta .
$$

Letting $\delta \rightarrow 0$, we find that

$$
C_{\alpha}\left(\left\{x: \limsup _{n \rightarrow \infty}\left|S_{R_{n}} \varphi(x)-\varphi(x)\right|>\eta\right\}\right)=0,
$$

for every $\eta>0$. The set of divergence is

$$
\bigcup_{k \geq 1}\left\{x: \limsup _{n \rightarrow \infty}\left|S_{R_{n}} \varphi(x)-\varphi(x)\right|>\frac{1}{k}\right\},
$$

which is a countable union of sets of $(\alpha, 2)$-capacity zero and so it also has $(\alpha, 2)$ capacity zero.

One consequence of this proposition is that the partial inverse Fourier integrals of functions in Sobolev classes $L_{\alpha}^{2}\left(\mathbb{R}^{d}\right)$ converge pointwise, with the possible exception of sets with zero $(\alpha-\varepsilon, 2)$-capacity, for every $\varepsilon>0$.

\section{REFERENCES}

1. G. Alexits, Convergence problems of orthogonal series, Translated from the German by I. Földer. International Series of Monographs in Pure and Applied Mathematics, Vol. 20, Pergamon Press, New York, 1961. MR0218827(36:1911)

2. Anthony Carbery, Dirk Gorges, Gianfranco Marletta, and Christoph Thiele, Convergence almost everywhere of certain partial sums of Fourier integrals, Bull. Lond. Math. Soc. 35 (2003), no. 2, 225-228. MR.1952399 (2003k:42027)

3. Anthony Carbery, José L. Rubio de Francia, and Luis Vega, Almost everywhere summability of Fourier integrals, J. London Math. Soc. (2) 38 (1988), no. 3, 513-524. MR0972135(90e:42033)

4. Anthony Carbery and Fernando Soria, Almost-everywhere convergence of Fourier integrals for functions in Sobolev spaces, and an $L^{2}$-localisation principle, Rev. Mat. Iberoamericana 4 (1988), no. 2, 319-337. MR1028744 (91d:42015)

5. Michael Christ, Javier Duoandikoetxea, and José L. Rubio de Francia, Maximal operators related to the Radon transform and the Calderón-Zygmund method of rotations, Duke Math. J. 53 (1986), no. 1, 189-209. MR0835805 (88d:42032)

6. Dirk Gorges, Convergence almost everywhere of partial sums of Fourier-integrals, Ph.D. thesis, Trier: Univ. Trier, Fachbereich IV, 50 p., 1998. MR.1952399 (2003k:42027)

7. Blin Mao, Heping Liu, and Shanzhen Lu, Norm inequality with power weights for a class of maximal spherical summation operators, J. Beijing Norm. Univ., Nat. Sci. 1989 (1989), no. 2, 1-4 (Chinese. English summary). MR1018436 (90h:42022)

8. Christopher Meaney, On almost-everywhere convergent eigenfunction expansions of the Laplace-Beltrami operator, Math. Proc. Cambridge Philos. Soc. 92 (1982), no. 1, 129-131. MR0662968 (83k:58093)

9. Emmanuel Montini, On the capacity of sets of divergence associated with the spherical partial integral operator, Trans. Amer. Math. Soc. 355 (2003), no. 4, 1415-1441 (electronic). MR.1946398 (2003h:42020) 
10. Elias M. Stein, Singular integrals and differentiability properties of functions, Princeton Mathematical Series, No. 30, Princeton University Press, Princeton, N.J., 1970. MR0290095 $(44: 7280)$

11. A. Zygmund, Trigonometric series. 2nd ed. Vols. I, II, Cambridge University Press, New York, 1959. MR0107776 (21:6498)

Dipartimento di Matematica e Applicazioni, Università di Milano Bicocca, Edificio U5, via Cozzi 53, 20125 Milano, Italy

E-mail address: leonardo@matapp.unimib.it

Department of Mathematics, Macquarie University, North Ryde NSW 2109, AusTRALIA

E-mail address: chrism@maths.mq.edu.au

Dipartimento di Matematica, Università di Roma "Tor Vergata", Via della Ricerca Scientifica, 00133 Roma, Italy

E-mail address: prestini@mat.uniroma2.it 